\title{
On the algebraic representation of selected optimal non-linear binary codes
}

\author{
Marcus Greferath and Jens Zumbrägel \\ Claude Shannon Institute \\ University College Dublin \\ Belfield, Dublin 4, Ireland \\ Email: \{marcus.greferath, jens.zumbragel\}@ucd.ie
}

\begin{abstract}
Revisiting an approach by Conway and Sloane we investigate a collection of optimal non-linear binary codes and represent them as (non-linear) codes over $\mathbb{Z}_{4}$. The Fourier transform will be used in order to analyze these codes, which leads to a new algebraic representation involving subgroups of the group of units in a certain ring.

One of our results is a new representation of Best's $(10,40,4)$ code as a coset of a subgroup in the group of invertible elements of the group ring $\mathbb{Z}_{4}\left[\mathbb{Z}_{5}\right]$. This yields a particularly simple algebraic decoding algorithm for this code.

The technique at hand is further applied to analyze Julin's $(12,144,4)$ code and the $(12,24,12)$ Hadamard code. It can also be used in order to construct a (non-optimal) binary $(14,56,6)$ code.
\end{abstract}

\section{INTRODUCTION}

Let $R$ be a finite ring and let $\delta$ be a metric on $R$, which is additively extended to a metric on $R^{n}$. An $(n, M, d)_{R, \delta}$ code is a (not necessarily linear) subset of $R^{n}$ having cardinality $M$ and minimum distance $d$ with respect to the metric $\delta$. Define $A_{R, \delta}(n, d)$ to be the maximum number $M$ such that an $(n, M, d)_{R, \delta}$ code over $R$ exists. A fundamental task in coding theory is to determine for given $n$ and $d$ the number $A_{R, \delta}(n, d)$, and to find a corresponding optimal code. In the following we consider the case $R=\mathbb{F}_{2}$ and $\delta=\delta_{H}$, the Hamming metric, or $R=\mathbb{Z}_{4}$ and $\delta=\delta_{\text {Lee }}$, the Lee metric. We will use the simplified notations $(n, M, d)$ for $(n, M, d)_{\mathbb{F}_{2}, \delta_{H}}$

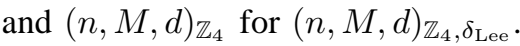

The purpose of this paper is to provide further insight into the algebraic representation of some optimal non-linear binary codes. Our work is based on the quaternary constructions for the Best code and the Julin code given by Conway and Sloane, in fact, we take the algebraic representation in [3] a step further in that we involve the multiplicative structure of the ambient group ring. More specifically, we use the Fourier transform as well as subgroups of the unit group of a group ring to analyze the codes.

As a result, we obtain a new succint description of Best's $(10,40,4)$ code as a subgroup coset in the unit group of the ring. This representation gives rise to a novel algebraic decoding algorithm for this code. We also apply this technique to analyze Julin's $(12,144,4)$ code and the $(12,24,12)$ Hadamard code. We further use it to construct a (non-optimal) binary $(14,56,6)$ code.

\section{Preliminaries}

\section{A. Codes over $\mathbb{Z}_{4}$}

In the beginning of the 90s several optimal non-linear binary codes were recognized as Gray images of $\mathbb{Z}_{4}$-linear codes (see, e.g., [6]). Since then codes over $\mathbb{Z}_{4}$ or over more general rings gained much attention in the literature.

Define the Lee weight on $\mathbb{Z}_{4}$ by

$$
w_{\text {Lee }}: \mathbb{Z}_{4} \rightarrow \mathbb{N}, \quad x \mapsto \min \{x, 4-x\} .
$$

Setting $\delta_{\text {Lee }}(x, y)=w_{\text {Lee }}(x-y)$, it turns out that $\left(\mathbb{Z}_{4}, \delta_{\text {Lee }}\right)$ is isometric to $\left(\mathbb{Z}_{2}^{2}, \delta_{H}\right)$ via the Gray mapping

$$
\mathbb{Z}_{4} \rightarrow \mathbb{Z}_{2}^{2}, \quad a+2 b \mapsto a(0,1)+b(1,1),
$$

where $a, b \in\{0,1\}$. The componentwise extension of this mapping to $\mathbb{Z}_{4}^{n}$ yields an isometry between $\left(\mathbb{Z}_{4}^{n}, \delta_{\text {Lee }}\right)$ and $\left(\mathbb{Z}_{2}^{2 n}, \delta_{H}\right)$.

We note that in order to view a (non-linear) binary code as a linear code over $\mathbb{Z}_{4}$ it is necessary that its cardinality is a power of 2 . Here we are interested in codes that do not satisfy this condition.

\section{B. Group rings and the Fourier transform}

A natural algebraic framework for cyclic codes is provided by group rings. For a finite ring $R$ the group ring $R\left[\mathbb{Z}_{n}\right]$ is the set of all $R$-valued functions on $\mathbb{Z}_{n}$, equipped with the natural addition and the multiplication $\star$ that is given by the cyclic convolution, i.e.,

$$
(f \star g)(j):=\sum_{i \in \mathbb{Z}_{n}} f(i) g(j-i),
$$

where $f, g \in R\left[\mathbb{Z}_{n}\right]$ and $j \in \mathbb{Z}_{n}$.

Letting $\delta_{j}$, for $j \in \mathbb{Z}_{n}$, be the element in $R\left[\mathbb{Z}_{n}\right]$ defined by $\delta_{j}(j)=1$ and $\delta_{j}(i)=0$ if $i \neq j$, we can write any element $f \in R\left[\mathbb{Z}_{n}\right]$ as a sum $\sum_{i \in \mathbb{Z}_{n}} f(i) \delta_{i}$. The element $f$ will also be denoted sometimes by a vector $(f(0), f(1), \ldots, f(n-1))$.

Using the concept of a group ring, a cyclic code of length $n$ over the ring $R$ can be understood as a subset in $R\left[\mathbb{Z}_{n}\right]$ that is closed under multiplication by the element $\delta_{1}$.

Now we define the discrete Fourier transform and state its fundamental properties (cf. [4]). 
Definition 1. Let $S \supseteq R$ be a ring extension that contains a primitive $n$-th root of unity $\omega$. For $f \in S\left[\mathbb{Z}_{n}\right]$ define the Fourier transform $\hat{f} \in S\left[\mathbb{Z}_{n}\right]$ by

$$
\hat{f}(i):=\sum_{j \in \mathbb{Z}_{n}} f(j) \omega^{-j i} .
$$

Proposition 2. For $f, g \in S\left[\mathbb{Z}_{n}\right]$ there holds

$$
(f \star g)^{\wedge}=\hat{f} \cdot \hat{g},
$$

where $\cdot$ denotes the componentwise product.

Proposition 3. Assume that $n \in R^{\times}$. Define the inverse transform by

$$
\tilde{f}(i):=\frac{1}{n} \sum_{j \in \mathbb{Z}_{n}} f(j) \omega^{i j} .
$$

Then it holds $\tilde{\hat{f}}=f=\hat{\tilde{f}}$ for all $f \in S\left[\mathbb{Z}_{n}\right]$.

We will further use the following results.

Lemma 4. For $f, g \in R\left[\mathbb{Z}_{n}\right]$ there holds

$$
\sum_{j \in \mathbb{Z}_{n}}(f \star g)(j)=\left(\sum_{i \in \mathbb{Z}_{n}} f(i)\right)\left(\sum_{j \in \mathbb{Z}_{n}} g(j)\right)
$$

Proof: We have $\sum_{j}(f \star g)(j)=\sum_{j} \sum_{i} f(i) g(j-i)=$ $\sum_{i} \sum_{j} f(i) g(j)=\left(\sum_{i} f(i)\right)\left(\sum_{j} g(j)\right)$.

Corollary 5. If $f \in R\left[\mathbb{Z}_{n}\right]$ is invertible then $\sum_{i} f(i) \in R^{\times}$.

Proof: If there exists $g \in R\left[\mathbb{Z}_{n}\right]$ with $f \star g=1$ then $1=\sum_{j}(f \star g)(j)=\left(\sum_{i} f(i)\right)\left(\sum_{j} g(j)\right)$, so that $\sum_{i} f(i)$ is invertible.

\section{ThE $(10,40,4)$ BEST CODE}

\section{A. The original binary code}

It is possible to prove $A_{\mathbb{F}_{2}, \delta_{H}}(10,4) \leq 40$ by a modification of the linear programming bound (see, e.g., [9, p. 541]). Best [1] came up with the construction of a binary code meeting this bound. This optimal binary $(10,40,4)$ code consists of the words

$$
\text { 0100000011, 0011111101, 1100101100, } 0001010111
$$

together with all cyclic shifts of these. The distance enumerator of Best's code is given by

$$
D_{H}(x, y)=x^{10}+22 x^{6} y^{4}+12 x^{4} y^{6}+5 x^{2} y^{8} .
$$

Its automorphism group is a semidirect product of the dihedral group $D_{5}$ and $\mathbb{Z}_{2}^{5}$ and hence has 320 elements. Litsyn and Vardy [8] showed that Best's code is unique, i.e., any binary $(10,40,4)$ code must be isometric to Best's code. Furthermore, it can be shown (cf. [1], [3]) that applying the so-called Construction A to Best's $(10,40,4)$ code yields the densest sphere packing known in 10 dimensions.
TABLE I

THE FOURIER TRANSFORM OF THE PENTACODE

The Fourier transform of the pentacode is given by the following vectors together with their negations.

$\left(1,3 \omega^{3}+3 \omega^{2}+3 \omega+2, \omega^{3}+3, \omega^{2}+3, \omega+3\right)$

$\left(1,3 \omega+1,3 \omega^{2}+1,3 \omega^{3}+1, \omega^{3}+\omega^{2}+\omega+2\right)$

$\left(1,3 \omega^{2}+\omega, \omega^{3}+2 \omega^{2}+\omega+1, \omega^{3}+3 \omega, 2 \omega^{3}+3 \omega^{2}+3 \omega+3\right)$

$\left(1,3 \omega^{3}+\omega^{2}, 3 \omega^{3}+3 \omega^{2}+2 \omega+3, \omega^{3}+\omega^{2}+2 \omega+1, \omega^{3}+3 \omega^{2}\right)$

$\left(1,2 \omega^{3}+\omega^{2}+\omega+1,3 \omega^{3}+\omega, 3 \omega^{3}+2 \omega^{2}+3 \omega+3, \omega^{2}+3 \omega\right)$

$\left(1, \omega^{3}+3 \omega^{2}+3 \omega, \omega^{3}+2 \omega+1,2 \omega^{3}+3 \omega^{2}+2 \omega+3,2 \omega^{2}+\omega+1\right)$

$\left(1,2 \omega^{3}+2 \omega^{2}+3 \omega+3,2 \omega^{3}+\omega^{2}+1, \omega^{3}+2 \omega^{2}+1,3 \omega^{3}+3 \omega^{2}+\omega\right)$

$\left(1, \omega^{2}+\omega+2,3 \omega^{3}+3 \omega+1, \omega^{3}+\omega+2,3 \omega^{2}+3 \omega+1\right)$

$\left(1, \omega^{3}+\omega^{2}+2 \omega, 3 \omega^{3}+\omega^{2}+3, \omega^{3}+3 \omega^{2}+3,3 \omega^{3}+3 \omega^{2}+2 \omega+2\right)$

$\left(1, \omega^{2}+3 \omega+3,3 \omega^{3}+2 \omega^{2}+3 \omega+2,3 \omega^{3}+\omega+3,2 \omega^{3}+\omega^{2}+\omega\right)$

$\left(1, \omega^{3}+\omega^{2}+3 \omega, 3 \omega^{3}+2 \omega^{2}+3,2 \omega^{3}+3 \omega^{2}+3,2 \omega^{3}+2 \omega^{2}+\omega+1\right)$

$\left(1,2 \omega^{2}+3 \omega+3,2 \omega^{3}+\omega^{2}+2 \omega+1,3 \omega^{3}+2 \omega+3,3 \omega^{3}+\omega^{2}+\omega\right)$

$\left(1,2 \omega^{3}+3 \omega^{2}+3 \omega, \omega^{3}+3 \omega+1, \omega^{3}+2 \omega^{2}+\omega+2,3 \omega^{2}+\omega+1\right)$

$\left(1, \omega^{3}+\omega^{2}+2 \omega+2,3 \omega^{3}+\omega^{2}+1, \omega^{3}+3 \omega^{2}+1,3 \omega^{3}+3 \omega^{2}+2 \omega\right)$

$\left(1, \omega^{2}+\omega+3,3 \omega^{3}+3 \omega+2, \omega^{3}+\omega+3,3 \omega^{2}+3 \omega+2\right)$

$\left(1,3 \omega^{3}+\omega^{2}+3 \omega+2,3 \omega^{3}+2 \omega^{2}+2 \omega+1, \omega^{2}+2 \omega+3,2 \omega^{3}+\omega+3\right)$

$\left(1,2 \omega^{3}+3 \omega+1,3 \omega^{2}+2 \omega+1, \omega^{3}+2 \omega^{2}+2 \omega+3, \omega^{3}+3 \omega^{2}+\omega+2\right)$

$\left(1,2 \omega^{3}+\omega^{2}+3 \omega+2,3 \omega^{3}+2 \omega^{2}+\omega+1, \omega^{3}+2 \omega^{2}+3 \omega, 2 \omega^{3}+3 \omega^{2}+\omega+3\right)$

$\left(1,3 \omega^{3}+\omega^{2}+2,3 \omega^{3}+3 \omega^{2}+2 \omega+1, \omega^{3}+\omega^{2}+2 \omega+3, \omega^{3}+3 \omega^{2}+2\right)$

$\left(1,2 \omega^{3}+\omega^{2}+3 \omega+1,3 \omega^{3}+2 \omega^{2}+\omega, \omega^{3}+2 \omega^{2}+3 \omega+3,2 \omega^{3}+3 \omega^{2}+\omega+2\right)$

\section{B. The pentacode over $\mathbb{Z}_{4}$}

The $(10,40,4)$ Best code cannot be recognized as a submodule over $\mathbb{Z}_{4}$, since the cardinality is not a power of 2 . Nevertheless, it was observed by Conway and Sloane [3] that the Best code has a sensible interpretation as a code over $\mathbb{Z}_{4}$. Namely, define a code $P \subseteq \mathbb{Z}_{4}^{5}$ consisting of all words

$$
(c-d, b, c, d, b+c) \quad \text { where } b, c, d \in\{1,3\}
$$

and all cyclic shifts of these. This code has parameters $(5,40,4)_{\mathbb{Z}_{4}}$ and is called the pentacode. It can be shown that the Gray image of $P$ is (up to equivalence) the $(10,40,4)$ code discovered by Best. Furthermore, the code $P$ is invariant under the automorphisms

$$
\begin{aligned}
(a, b, c, d, e) & \mapsto(-a,-b,-c,-d,-e), \\
(a, b, c, d, e) & \mapsto(-a, 2-b, c, 2-d,-e), \\
(a, b, c, d, e) & \mapsto(b, c, d, e, a), \\
(a, b, c, d, e) & \mapsto(2+e, 2+d, 2+c, 2+b, 2+a) .
\end{aligned}
$$

\section{Spectral analysis of the pentacode}

In order to apply the Fourier transform, we chose to analyze the pentacode, because Best's original binary code does not satisfy $n \in \mathbb{F}_{2}^{\times}$. For this we find that the Galois ring $\operatorname{GR}(4,4)$ as an extension of $\mathbb{Z}_{4}$ contains the required primitive 5 -th root of unity $\omega$. The minimal polynomial of $\omega$ over $\mathbb{Z}_{4}$ is given by

$$
\varphi_{\omega}=x^{4}+x^{3}+x^{2}+x+1 .
$$

We computed the Fourier transform of all words of the pentacode. The result is shown in Table I] It is apparent that the spectrum of each word of $P$ solely consists of invertible elements in $\operatorname{GR}(4,4)$. We can further make the following 
observation. Let

$$
\begin{aligned}
& \hat{f}:=\left(1,3 \omega+1,3 \omega^{2}+1,3 \omega^{3}+1,3 \omega^{4}+1\right) \\
& \hat{g}:=\left(1,3 \omega+2,3 \omega^{2}+2,3 \omega^{3}+2,3 \omega^{4}+2\right) \\
& \hat{h}:=\left(1,2 \omega+3,2 \omega^{2}+3,2 \omega^{3}+3,2 \omega^{4}+3\right)
\end{aligned}
$$

Then for each word $c \in P$ there holds

$$
\hat{c}=\hat{f} \cdot(-1)^{i} \cdot \hat{h}^{j} \cdot \hat{g}^{k} \quad \text { for some } i, j \in \mathbb{Z}_{2}, k \in \mathbb{Z}_{10} .
$$

\section{Algebraic representation}

The preceeding observations lead to the following algebraic structure of the pentacode. By applying the inverse transform and doing some rescaling we obtain the vectors

$$
\begin{aligned}
f & =(1,1,1,2,0), \\
g & =(2,1,0,0,0), \\
h & =(1,2,0,0,0) .
\end{aligned}
$$

Here $h^{2}=1$ and $g$ is of order 10 . This yields the following theorem:

Theorem 6. Let $f, g, h \in \mathbb{Z}_{4}\left[\mathbb{Z}_{5}\right]$ be as above. For each word $c$ of the pentacode $P$ there holds

$$
c=f \star(-1)^{i} \star h^{j} \star g^{k}
$$

for some $i, j \in \mathbb{Z}_{2}$ and $k \in \mathbb{Z}_{10}$. Consequently, the pentacode is a coset

$$
P=f \star U,
$$

where $U$ is a 40 element subgroup of the group of invertible elements of the group ring $S:=\mathbb{Z}_{4}\left[\mathbb{Z}_{5}\right]$.

One can prove that the ring $S=\mathbb{Z}_{4}\left[\mathbb{Z}_{5}\right]$ is isomorphic to $\operatorname{GR}(4,4) \times \mathbb{Z}_{4}$, and hence the unit group $S^{\times}$is isomorphic to $\mathbb{Z}_{2}^{4} \times \mathbb{Z}_{15} \times \mathbb{Z}_{2} \cong \mathbb{Z}_{2}^{5} \times \mathbb{Z}_{3} \times \mathbb{Z}_{5}$ (see [10]). From this it is easy to see that there are $\left(\begin{array}{l}5 \\ 3\end{array}\right)_{2}=155$ subgroups $U$ of order 40 in the unit group of $S$; here $\left(\begin{array}{l}5 \\ 3\end{array}\right)_{2}$ denotes the Gaussian binomial coefficient, i.e., the number of 3-dimensional subspaces of $\mathbb{Z}_{2}^{5}$. By a computer search we found that only 2 of these subgroups yield (up to equivalence) the pentacode. Moreover, the pentacode occurs twice among the 12 cosets of each of these two subgroups.

The four algebraic representations of the pentacode as $f \star U$, where $f \in S$ and $U$ is a subgroup of $S^{\times}$, are given in Table III In each case, we state four generators of the subgroup $U$, which have order $5,2,2$, and 2, respectively, and we chose the representative $f$ to be of minimal degree in the 'polynomial' representation $\sum_{i \in \mathbb{Z}_{j}} f(i) \delta_{1}^{i}$. The original pentacode $P$ of Theorem 6 is found as $f_{1,1} \star U_{1}$; for this, note that $g^{5}=(1,0,0,0,2)$ and $g^{6}=(0,1,0,0,0)$.

For each subgroup $U_{j}, j=1,2$, the pentacodes $f_{j, 1} \star U_{j}$ and $f_{j, 2} \star U_{j}$ are related as $f_{j, 2} \star U_{j}=a \star f_{j, 1} \star U_{j}$, where $a=(3,2,2,2,2) \notin U_{j}$ is a unit element; this element has the property that the left multiplication

$$
L_{a}: S^{\times} \rightarrow S^{\times}, \quad x \mapsto a \star x
$$

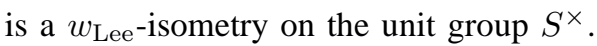

TABLE II

THE FOUR REPRESENTATIONS OF THE PENTACODE AS $f \star U$

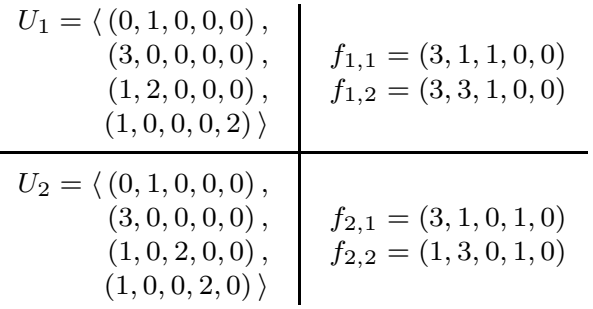

In fact, the set of $s \in S$ such that $L_{s}: S^{\times} \rightarrow S^{\times}$is an isometry of the unit group $S^{\times}$equals $\left\langle\delta_{1},-1, a\right\rangle$, whereas the set of $s \in S$ such that $L_{s}: C \rightarrow C$ is a code isometry equals $\left\langle\delta_{1},-1\right\rangle$, for all four pentacodes $C$. In particular, not all left multiplications of elements in $U_{j}$ are isometries.

We note that [3] mentions also four pentacodes $\mathcal{B}_{0}, \mathcal{B}_{1}, \mathcal{B}_{2}$, $\mathcal{B}_{3}$, which are constructed as orbits of a group of isometries on $\mathbb{Z}_{4}^{5}$. Here, $\mathcal{B}_{0}$ equals $f_{1,1} \star U_{1}$ and $\mathcal{B}_{2}$ equals $f_{1,2} \star U_{1}$, whereas $\mathcal{B}_{1}$ and $\mathcal{B}_{3}$ are different from $f_{2,1} \star U_{2}$ and $f_{2,2} \star U_{2}$.

\section{E. A decoding algorithm}

We present a simple decoding algorithm for the pentacode which is based on its algebraic representation as $f \star U$ in the group ring. The decoding algorithm differs considerably from the one given in [3].

In the following we will write $f g$ for the convolution $f \star g$ in a group ring. For concreteness we consider in the ring $S=\mathbb{Z}_{4}\left[\mathbb{Z}_{5}\right]$ the pentacode $C=f_{2,1} U_{2}=f U$, with $f=(3,3,2,1,0)=(0,1,1,1,2)^{-1} \in C$ and $U=$ $U_{2}=\left\langle\delta_{1},-1, g, h\right\rangle$, where $g=(1,0,2,0,0)=2 \delta_{2}+1$ and $h=(1,0,0,2,0)=2 \delta_{3}+1$. Hence $C$ is given as

$$
C=\left\{f(-1)^{i} g^{j} h^{k} \delta_{\ell} \mid i, j, k \in \mathbb{Z}_{2}, \ell \in \mathbb{Z}_{5}\right\} .
$$

The following lemma gives a simple membership test for $U$.

Lemma 7. Let $s \in S$. Then $s \in U$ if and only if

$$
s \equiv \delta_{\ell} \bmod 2 \quad \text { and } \quad \delta_{-\ell} s=2\left(i+j \delta_{2}+k \delta_{3}\right)+1
$$

for some $\ell \in \mathbb{Z}_{5}$ and $i, j, k \in \mathbb{Z}_{2}$.

Proof: An element $u \in U$ is of the form $(-1)^{i} g^{j} h^{k} \delta_{\ell}=$ $(2+1)^{i}\left(2 \delta_{2}+1\right)^{j}\left(2 \delta_{3}+1\right)^{k} \delta_{\ell}=\left(2\left(i+j \delta_{2}+k \delta_{3}\right)+1\right) \delta_{\ell}$, for some $\ell \in \mathbb{Z}_{5}$ and $i, j, k \in \mathbb{Z}_{2}$.

Given $y \in S$ we can check whether $y \in C=f U$ by applying the test of Lemma 7 to $z=f^{-1} y$. In case $y \in C$ we also obtain this way the 'message' $(i, j, k, \ell) \in \mathbb{Z}_{2}^{3} \times \mathbb{Z}_{5}$.

Now we present a decoding algorithm for the pentacode $C$. Let $y \in S$ be the received vector, and assume that $y=c+e=$ $f u+e$, where $c \in C, u \in U$, and $e \in S$ is an error vector of Lee weight $\leq 2$. We note that $f^{-1}=(0,1,1,1,2)$.

1) Compute $z:=f^{-1} y$.

[In fact, $z=u+f^{-1} e$. Note that $u \equiv \delta_{\ell} \bmod 2$, so that $w_{H}(u \bmod 2)=1$. Furthermore, $w_{H}\left(f^{-1} e \bmod 2\right)$ is odd if and only if $w_{H}(e \bmod 2)$ is odd.] 
2a) Case $w_{H}(z \bmod 2)$ odd: Check if $z \in U$ by applying Lemma 7 If yes, conclude that no error occurred, and output the corresponding message $(i, j, k, \ell)$; if no, conclude that two errors occurred.

[Note that $w_{H}(e \bmod 2)$ is even.]

2b) Case $w_{H}(z \bmod 2)$ even:

[Note that $w_{H}(e \bmod 2)$ is odd, and hence $e= \pm \delta_{r}$ for some $r \in \mathbb{Z}_{5}$. We thus have $z=u \pm \delta_{r} f^{-1}$, where $u=\delta_{\ell}\left(2\left(i+j \delta_{2}+k \delta_{3}\right)+1\right)$ for some $\ell \in \mathbb{Z}_{5}$ and $i, j, k \in \mathbb{Z}_{2}$.]

Determine $\ell \in \mathbb{Z}_{5}, r \in \mathbb{Z}_{5}$, and a sign according to the following table (with $m \in \mathbb{Z}_{5}$ to be chosen):

\begin{tabular}{c|c}
$\delta_{m} z$ & $(m+\ell, m+r, \pm)$ \\
\hline$(o, \pm 1, o, o, 2)$ & $(0,0, \pm)$ \\
$(o, o, \pm 1, o, 0)$ & $(3,4, \pm)$ \\
$(o, \pm 1, e, 2, e)$ & $(2,4, \pm)$ \\
$( \pm 1, o, e, 0, e)$ & $(4,3, \pm)$ \\
$( \pm 1, e, \pm 1, e, e)$ & $(1,4, \pm)$
\end{tabular}

Here $e$ stands for an even element and $o$ for an odd element of $\mathbb{Z}_{4}$.

[Note that $f^{-1}=(0,1,1,1,2)$ and $2\left(i+j \delta_{2}+k \delta_{3}\right)+1$ is of the form $(o, 0, e, e, 0)$.]

Then $\delta_{-\ell}\left(z \mp \delta_{r} f^{-1}\right)=2\left(i+j \delta_{2}+k \delta_{3}\right)+1$ for some $i, j, k \in \mathbb{Z}_{2}$; output the message $(i, j, k, l)$.

It is not hard to see that the pattern given in the table determine uniquely $\ell \in \mathbb{Z}_{5}, r \in \mathbb{Z}_{5}$, and the sign of the error $e= \pm \delta_{r}$. Thus, the decoding algorithm corrects all errors of Lee weight 1 and detects all errors of Lee weight 2. This shows again that the code has minimum Lee distance 4 .

\section{INVESTIGATION OF OTHER CODES}

The Gray images of $\mathbb{Z}_{4}$-codes of the form $f \star U$, where $f \in$ $S=\mathbb{Z}_{4}\left[\mathbb{Z}_{n}\right]$ and $U$ is a subgroup of the unit group $S^{\times}$, yield good binary codes in many cases. For example, binary codes with parameters $(6,4,4),(8,16,4),(10,40,4),(12,128,4)$, $(14,392,4),(12,24,6)$, and $(14,56,6)$ can be constructed this way. In this section we study some of these cases as well as the $(12,144,4)$ Julin code in greater detail. We start with a preliminary observation.

Proposition 8. Let $S:=\mathbb{Z}_{4}\left[\mathbb{Z}_{n}\right]$, let $f \in S$ and $U \subseteq S^{\times}$, and let $C:=f \star U$. Then the Lee distance between two codewords of $C$ is even, in particular the minimum Lee distance of the code $C$ is even.

Proof: Let $S_{\text {odd }}:=\left\{f \in S \mid \sum_{i} f(i) \in \mathbb{Z}_{4}^{\times}=\{1,3\}\right\}$ and let $S_{\text {even }}:=S \backslash S_{\text {odd }}$. By Corollary $5, U \subseteq S^{\times} \subseteq S_{\text {odd }}$.

Case 1: $f \in S_{\text {odd }}$. Then from Lemma 4 it follows $C=$ $f \star U \subseteq S_{\text {odd }}$. Let $c, c^{\prime} \in C \subseteq S_{\text {odd }}$. Then $\sum_{i}\left(c-c^{\prime}\right)(i)=$ $\sum_{i} c(i)-\sum_{i} c^{\prime}(i)$ is even, so that $c-c^{\prime} \in S_{\text {even. }}$. It follows that $w_{\text {Lee }}\left(c-c^{\prime}\right)=\sum_{i} w_{\text {Lee }}\left(c-c^{\prime}\right)(i)$ is even.

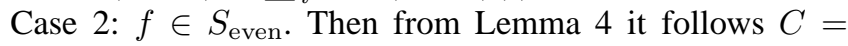
$f \star U \subseteq S_{\text {even }}$. As before, for $c, c^{\prime} \in C$ it follows $c-c^{\prime} \in S_{\text {even }}$ and thus $w_{\text {Lee }}\left(c-c^{\prime}\right)$ is even.

\section{A. The $(12,144,4)$ Julin code}

Julin's binary $(12,144,4)$ code [7] (see also [9, p. 70f]) can be constructed by taking as words the 132 blocks of a Steiner $S(5,6,12)$ system and adding six words each of weight 2 and 10. The code was shown to be optimal in [11]. Conway and Sloane [3] provided a quaternary construction for a canonical version of the Julin code. In this construction one defines the vectors

$$
\begin{aligned}
& c_{1}=(0,0,1,1,2,2) \quad c_{2}=(0,0,2,2,1,1) \\
& c_{3}=(0,1,0,2,1,2) \quad c_{4}=(0,2,0,1,2,1) \\
& c_{5}=(0,1,2,0,2,1) \quad c_{6}=(0,1,1,3,3,2) \\
& c_{7}=(0,1,2,3,1,3) \quad c_{8}=(0,1,3,1,2,3) \\
& c_{9}=(0,1,3,2,3,1) \quad c_{10}=(0,2,1,1,3,3) \\
& c_{11}=(3,1, \ldots, 1) \quad c_{12}=(2,0, \ldots, 0) \quad c_{13}=(0,2, \ldots, 2)
\end{aligned}
$$

and let the quaternary Julin code $J$ consist of all cyclic shifts and negations of the vectors $c_{1}, \ldots, c_{13}$. This code has parameters $(6,144,4)_{\mathbb{Z}_{4}}$.

We performed a spectral analysis of the quaternary Julin code $J$. Although $n=6$ is not a unit in characteristic 4 , the Fourier transform provided us with enough information to find the following algebraic representation.

Let $S$ be the group ring $\mathbb{Z}_{4}\left[\mathbb{Z}_{6}\right]$, and let $g=$ $(0,1,1,1,1,1) \in S$ which is an invertible element of order 2 . We consider the subgroup $U$ of $S^{\times}$generated by $-1, \delta_{1}$, and $g$, which has 24 elements.

Then $J$ is invariant under the group $U$; it is actually a union of 8 cosets of $U$. More concretely, $J$ can be written as

$$
\begin{aligned}
J=\{ & \left.c_{1}, c_{5}\right\} \star(\{1,(0,0,3,1,0,1)\} \star U) \\
& \cup c_{5} \star(\{(0,1,0,1,0,3),(1,0,0,0,1,3)\} \star U) \\
& \cup c_{1} \star(0,0,0,1,1,1) \star U \\
& \cup c_{11} \star U .
\end{aligned}
$$

\section{B. The $(12,24,6)$ Hadamard code}

The binary $(12,24,6)$ binary Hadamard code (see, e.g., [9, p. 49]) is constructed from a Hadamard matrix $H$ of order 12, and such a matrix can be obtained from the Paley construction. The Hadamard matrix $H$ is changed into a binary matrix $A$, where $+1 \mathrm{~s}$ are replaced by $0 \mathrm{~s}$ and $-1 \mathrm{~s}$ by $1 \mathrm{~s}$. Then the Hadamard code consists of all rows of the matrix $A$, together with their complements. From the definition of Hadamard matrix the code has parameters $(12,24,6)$, which can be seen to be optimal by applying the Plotkin bound (see [9, p. 43]).

We now give an algebraic representation of a quaternary $(6,24,6)_{\mathbb{Z}_{4}}$ by using again the group ring $S=\mathbb{Z}_{4}\left[\mathbb{Z}_{6}\right]$. Let $U$ be the subgroup of $S^{\times}$generated by the elements $(2,1,1,1,1,1),(0,3,3,3,3,3)$, and $(2,0,0,3,0,0)$ of order 2 , as well as the element $(0,0,1,0,0,0)$ of order 3 . Then $U$ has 24 elements, and

$$
C=f \star U
$$

with $f=(0,1,2,0,0,0)$ defines a $(6,24,6)_{\mathbb{Z}_{4}}$ code. It can be shown that the Gray map of $C$ is a translation of a $(12,24,6)$ Hadamard code. 
We remark that a binary $(12,24,6)$ code was not found in a similar way in the binary group ring $\mathbb{Z}_{2}\left[\mathbb{Z}_{12}\right]$.

C. $A(14,56,6)$ code

We performed a computer search to obtain an interesting heptacode. For this we took a similar avenue as for the Best code and define:

$$
\begin{aligned}
& f=(1,2,3,1,1,0,0) \\
& g=(2,1,0,0,0,0,0) \\
& h=(1,2,0,0,0,0,0)
\end{aligned}
$$

Again $h^{2}=1$, and here $g$ is of order 14 . We form the code

$$
H:=\left\{f \star(-1)^{i} \star h^{j} \star g^{k} \mid i, j \in \mathbb{Z}_{2}, k \in \mathbb{Z}_{14}\right\},
$$

which has 56 words and is of minimum Lee distance 6 . The code turns out to be distance-invariant and its distance enumerator is given by

$$
D_{\text {Lee }}(x, y)=x^{14}+36 x^{8} y^{6}+7 x^{6} y^{8}+12 x^{4} y^{10} .
$$

The Gray image of $H$ is a binary $(14,56,6)$ code. Consulting Grassl's tables [5] we find that for a linear binary code only the parameters $\left(14,2^{5}, 6\right)$ are possible. From Brouwer's table [2] however we find a strong competitor, namely the doubly shortened Nordstrom-Robinson code, which is a $(14,64,6)$ code.

We remark that by shortening the code $H$ we get a code with parameters $(13,28,6)$, which are the same as for the socalled conference matrix code of length 13 (see [9, p. 57]).

\section{CONCLUSIONS}

In this paper we provided new insights into the algebraic representation of Best's $(10,40,4)$ code and other non-linear binary codes. We established the discrete Fourier transform as a useful tool when analyzing non-linear codes, and found a presentation of certain optimal non-linear binary codes as coset(s) of a subgroup in the unit group of a ring. It is an open problem whether asymptotically good codes can be obtained by this method.

\section{ACKNOWLEDGEMENTS}

The authors would like to thank Michael Kiermaier and Oliver Gnilke for inspiring discussions. This work was supported in part by Science Foundation Ireland, Grant 06/MI/006 and Grant 08/IN.1/I1950.

\section{REFERENCES}

[1] M. R. Best, "Binary Codes with a Minimum Distance of Four," IEEE Trans. Inform. Theory, vol. 26, pp. 738-742, 1980.

[2] A.E. Brouwer, Table of general binary codes. Available at http://www.win.tue.nl/ aeb/codes/binary-1.html

[3] J.H. Conway and N.J.A. Sloane, "Quaternary Constructions for the Binary Single-Error-Correcting Codes of Julin, Best and Others," Designs, Codes and Cryptography, vol. 4, pp. 31-42, 1994.

[4] E. Dubois and A. N. Venetsanopoulos, "The Discrete Fourier Transform Over Finite Rings with Application to Fast Convolution," IEEE Trans. Computers, vol. C-27, pp. 586-593, 1978.

[5] M. Grassl, Code Tables: Bounds on the parameters of various types of codes. Available at http://www.codetables.de/

[6] A. R. Hammons, P. V. Kumar, P. Vijay, A. R. Calderbank, N. J. A. Sloane, and P. Solé, "The $\mathbb{Z}_{4}$-linearity of Kerdock, Preparata, Goethals, and related codes," IEEE Trans. Inform. Theory, vol. 40, pp. 301-319, 1994.

[7] D. Julin, "Two Improved Block Codes." IRE Trans. Inform. Theory, vol. 11, p. 459, 1965.

[8] S. Litsyn and A. Vardy, "The Uniqueness of the Best Code," IEEE Trans. Inform. Theory, vol. 40, pp. 1693-1698, 1994.

[9] F. J. MacWilliams and N. J. A. Sloane, The Theory of Error-CorrectingCodes. Amsterdam, The Netherlands: North-Holland, 1978.

[10] B. R. McDonald, Finite rings with identity. New York, USA: Marcel Dekker Inc., 1974.

[11] P. R. J. Ostergard, T. Baicheva, E. Kolev, "Optimal binary one-errorcorrecting codes of length 10 have 72 codewords," IEEE Trans. Inform. Theory, vol. 45, pp. 1229-1231, 1999. 